\title{
CONTABILIDAD MACROECONÓMICA Y DESARROLLO REGIONAL
}

\author{
Mg. Gilberto José Rafael Cárdenas Núñez*
}

\begin{abstract}
RESUMEN
Las cuentas de producción departamental actualizadas son indispensables para los planes de desarrollo que orienten el crecimiento de la economía nacional y el desarrollo integral del país. Esta información tiene que elaborarse en forma departamental.

Las enormes diferencias productivas y de bienestar entre los departamentos del país obligan al sector público y al privado a trabajar en forma conjunta para realizar las inversiones necesarias en base al capital humano y los recursos naturales disponibles actualmente en el país.
\end{abstract}

\section{INTRODUCCIÓN}

Los grandes movimientos regionales y departamentales ocurridos en el período 2001 - 2002 a lo largo y ancho del país, empezando por Iquitos (Loreto) y recientemente en Tarapoto (San Martín) son una clara manifestación del enorme impulso reformista de las poblaciones de provincias y del interior del país; son expresiones reformistas debido a que la población de lugares alejados de Lima Metropolitana ansían canalizar su interés y búsqueda del crecimiento y desarrollo económico pero descentralizado para el Perú.

En el presente trabajo se exponen las vinculaciones entre la información y el desarrollo económico que supone un plan o programa de desarrollo nacional, regional - departamental con el fin de canalizar la inquietud o preocupación por el progreso y bienestar en el interior del país, partiendo de conocer y analizar las cuentas nacionales respectivas.

\section{LA SITUACIÓN ACTUAL: LA CUENTA DEL PRODUCTO BRUTO INTERNO (PBI)}

La información estadística del PBI y su generación en los diferentes departamentos del país han sido elaboradas y publicadas por diversas instituciones especializadas, como es el caso del Instituto Nacional de Estadística e Informática ${ }^{1}$ (INEI) por parte del sector público, y del Instituto CUANTO ${ }^{2}$ por parte del sector no público, que con algunas pequeñas diferencias entre sus datos, coinciden en lo principal: demuestran la gran concentración productiva estructural de bienes y servicios de Lima con respecto al país.

○

La Gaceta. Boletín del Congreso de la República. suplemento del diario La República, del 11 de agosto del 2002 , ma-Perú.

Descifra; Secretaría Técnica para el Proceso de Descentralización del Ministerio de la Presidencia, N. a 2, Junio 2002, Limä-Perú. 
Cabe indicar que Lima Metropolitana concentra lo principal de la producción manufacturera en lo que se refiere a bienes, $y$ de los servicios en lo que se refiere a comercio, educación, comunicaciones y otros. Las provincias del interior son las grandes proveedoras de bienes de origen agropecuario, minero, pesquero y forestal de la economía peruana.
El INEI presenta información estadística del PBI en el departamento de Lima con un nivel de $52.7 \%$ con respecto al $\mathrm{PBI}$ nacional al 2001, mientras que CUANTO le da a Lima el $47.4 \%$ para el año 2000 , tal como podemos ver en el cuadro siguiente:

PRODUCTO BRUTO INTERNO POR DEPARTAMENTO Millones de nuevos soles a precios corrientes

\begin{tabular}{|c|c|c|c|c|c|c|c|c|c|c|}
\hline \multirow[b]{2}{*}{ DEPARTAMENTO } & \multicolumn{10}{|c|}{ AÑO Y PORCENTAJE } \\
\hline & 1996 & $\%$ & 1997 & $\%$ & 1998 & $\%$ & 1999 & $\%$ & 2000 & $\%$ \\
\hline AMAZONAS & 1,547 & 1.1 & 1,669 & 1.1 & 1,780 & 1.1 & 1,541 & 0.9 & 1,673 & 0.9 \\
\hline ANCASH & 3,442 & 2.5 & 3,851 & 2.4 & 4,089 & 2.5 & 4,883 & 2.8 & 5,567 & 3.0 \\
\hline APURIMAC & 1,076 & 0.8 & 1,071 & 0.7 & 1,083 & 0.7 & 1,178 & 0.7 & 1,108 & 0.6 \\
\hline AREQUIPA & 9,117 & 6.7 & 10,356 & 6.6 & 10,885 & 6.5 & 11,123 & 6.4 & 12,021 & 6.4 \\
\hline AYACUCHO & 1,540 & 1.1 & 1,686 & 1.1 & 1,769 & 1.1 & 1,738 & 1.0 & 1,881 & 1.0 \\
\hline CAJAMARCA & 3,216 & 2.3 & 3,668 & 2.3 & 4,291 & 2.6 & 4,654 & 2.7 & 5,842 & 3.1 \\
\hline CUSCO & 3,473 & 2.5 & 3,931 & 2.5 & 4,298 & 2.6 & 4,376 & 2.5 & 4,781 & 2.6 \\
\hline HUANCAVELICA & 1,242 & 0.9 & 1,327 & 0.8 & 1,346 & 0.8 & 1,382 & 0.8 & 1,523 & 0.8 \\
\hline HUÁNUCO & 2,474 & 1.8 & 2,677 & 1.7 & 2,766 & 1.7 & 2,817 & 1.6 & 3,160 & 1.7 \\
\hline ICA & 4,193 & 3.1 & 4,791 & 3.0 & 4,914 & 3.0 & 5,161 & 3.0 & 5,549 & 3.0 \\
\hline UNÍN & 6,363 & 4.6 & 7,275 & 4.6 & 7,733 & 4.6 & 7,798 & 4.5 & 8,394 & 4.5 \\
\hline LA LIBERTAD & 7,894 & 5.8 & 8.848 & 5.6 & 9,773 & 5.9 & 9,798 & 5.6 & 10,828 & 5.8 \\
\hline LAMBAYEQUE & 5,973 & 4.4 & 6,378 & 4.1 & 6,874 & 4.1 & 7,046 & 4.0 & 7,644 & 4.1 \\
\hline LIMA & 60,952 & 44.5 & 73,558 & 46.8 & 77,766 & 46.7 & 82,700 & 47.3 & 88,450 & 47.4 \\
\hline LORETO & 4,304 & 3.1 & 4,777 & 3.0 & 5,215 & 3.1 & 5,495 & 3.1 & 5,865 & 3.1 \\
\hline MADRE DE DIOS & 641 & 0.5 & 559 & 0.4 & 565 & 0.3 & 613 & 0.4 & 379 & 0.2 \\
\hline MOQUEGUA & 3,158 & 2.3 & 3,553 & 2.3 & 3,554 & 2.1 & 3,777 & 2.2 & 3,256 & 1.7 \\
\hline PASCO & 1,907 & 1.4 & 1,968 & 1.3 & 1,950 & 1.2 & 2.089 & 1.2 & 1.154 & 0.6 \\
\hline PIURA & 5,514 & 4.0 & 5,786 & 3.7 & 5,469 & 3.3 & 5,800 & 3.3 & 6,289 & 3.4 \\
\hline PUNO & 2,595 & 1.9 & 2,842 & 1.8 & 3,082 & 1.9 & 3,224 & 1.8 & 3,299 & 1.8 \\
\hline SAN MARTÍN & 2,040 & 1.5 & 2,287 & 1.5 & 2,586 & 1.6 & 2,610 & 1.5 & 2,793 & 1.5 \\
\hline TACNA & 2,182 & 1.6 & 2,106 & 1.3 & 2,138 & 1.3 & 2,253 & 1.3 & 2,563 & 1.4 \\
\hline TUMBES & 601 & 0.4 & 740 & 0.5 & 658 & 0.4 & 759 & 0.4 & 861 & 0.5 \\
\hline UCAYALI & 1,575 & 1.2 & 1,568 & 1.0 & 1,932 & 1.2 & 1,904 & 1.1 & 1,878 & 1.0 \\
\hline TOTAL & 136,929 & 100 & 157,274 & 100 & 166,514 & 100 & 174,719 & 100 & 186,756 & 100 \\
\hline
\end{tabular}

FUENTE: CUANTO

Una lectura dedicada del cuadro expuesto indica que aproximadamente la mitad de la generación de producto en el Perú se realiza en el departamento de Lima. Lejos le sigue el departamento de Arequipa, con el $6.4 \%$, La Libertad con $5.8 \%$, Junín con $4.5 \%$ y Lambayeque con $4.1 \%$.

En el extremo opuesto se encuentran los departamentos de Madre de Dios con $0.2 \%$ del PBI nacional, Tumbes con $0.5 \%$,
Apurímac con $0.6 \%$, Pasco con $0.6 \%$, Huancavelica con $0.8 \%$ y Amazonas con $0.9 \%$.

Se puede apreciar que la producción se genera precisamente en el departamento que posee pocos recursos naturales, como lo es Lima, y es muy pequeña, aunque con variaciones, la participación de los departamentos en donde los recursos naturales son abundantes. 
CUENTA DE RECURSOS HUMANOS: LA POBLACIÓN

Esta concentración de riqueza nacional en Lima está estrechamente relacionada con el hecho de que el mencionado departamento concentra también la mayor parte de la población nacional, proyectado al 2000 , con 7`466,190 habitantes ${ }^{3}$. Le siguen en población los departamentos de Piura con 1'545,771 habitantes, La Libertad con 1'465,970, Cajamarca con 1'411,942, y Puno con 1'199,398 habitantes. En el otro extremo, poco poblados, se encuentran los departamentos de Madre de Dios con sólo 84,383 habitantes, Tumbes con 193,840 , Moquegua con 147,374 y Tacna con 277,188 .

La densidad poblacional (habitantes por kilómetro cuadrado) le dan a la Provincia Constitucional del Callao un índice de 5,264; 214.5 al Departamento de Lima, 76.8 a Lambayeque, 57.5 a La Libertad, 43.1 a Piura, 42.4 a Cajamarca y 41.5 a Tumbes.

Es notable que la población se concentre cada vez más en Lima y el Callao en desmedro de las provincias. Así, la densidad poblacional en el departamento de Madre de Dios es 1.0, 2.4 en Loreto, 4.1 en Ucayali, 9.4 en Moquegua y 9,8 en Pasco; o sea, vastos territorios plenos de recursos están despoblados y áreas pequeñas están superpobladas.

\section{LA PRODUCCIÓN POR HABITANTE A NIVEL DEPARTAMENTAL: PRO- DUCTIVIDADES}

Relacionando el PBI con la población se elabora un indicador de nivel de productividad que nos indica los niveles reales de producción por persona en términos monetarios promedio en un período determinado. Algunos de los resultados indican que en el departamento de Lima cada habitante genera anualmente $11,864.7$ millones de soles promedio; 11,203.6 en Arequipa, 7,386.2 y en La Libertad, para mencionar los primeros.

Entre los departamentos más alejados de la capital se encuentran los siguientes resultados: Apurímac con 2,595.4 millones de soles anuales promedio por habitante, Puno con 2,750.5, Huancavelica con $3,532.9$, Ayacucho con 3,566, Tumbes con $4,441.8$, Loreto con $6,661.2$ y Tacna con 9,246.4.

Este indicador da una idea de la productividad comparada entre los habitantes de un departamento y de otro. La relación entre las productividades de Lima o Arequipa y Apurímac o Puno es superior a 4 veces. Esta relación tan grande entre las productividades permite vislumbrar los diferenciados niveles de inversión y avance tecnológico y productivo entre los departamentos del mismo país. Esto sin contar con enorme diferenciación intra departamental.

\section{CUENTAS Y PLANES}

Hace aproximadamente 10 años, en 1992, el primer gobierno de Alberto Fujimori decidió disolver el Instituto Nacional de Planificación del Perú. Desde entonces los planes de desarrollo departamentales, regionales o nacionales dejaron un espacio técnico sin ocupar sino hasta hace poco.

Sin embargo, la necesidad de los planes era y es apremiante debido al interés público y no público de contar con instrumentos que les permitan ampliar sus horizontes y perspectivas de desarrollo. Recientemente, la Secretaría Técnica para el Proceso de Descentralización ha publicado dos documentos de importancia para tratar de

INEI. Perú: Compendio Estadístico 2001, Lima, junio del 2001. 
subsanar el vacío creado por el Gobierno de Fujimori. En primer lugar, planes referidos al desarrollo departamental ${ }^{4}$ y el documento de nivel nacional ${ }^{5}$, los cuales permiten comprender la importancia de las propuestas para el desarrollo nacional y departamental, así como sus vinculaciones y conflictos.

Las cuentas nacionales tienen la especial importancia de mirar al Perú como en una gran fotografía de la situación en un momento dado. Pero es el estudio de la dinámica económica, social, política y cultural lo que permite comprender las grandes dificultades y retos que tiene el crecimiento y desarrollo nacionales, y son los planes los instrumentos que permiten diseñar las estrategias para superarlas.

Los grandes retos contemporáneos que presentan los planes mencionados para el desarrollo nacional, macrorregional $y$ departamental en el país, desde el punto de vista económico, y que están en actual debate, se pueden resumir de la siguiente manera:

1. Severa restricción del financiamiento externo.

2. Estrechez de los recursos fiscales.

3. Dificultad para reactivar la producción en forma sostenida y generalizada y su relación con la dinámica internacional de los bloques económicos.

4. Grandes brechas en la distribución del ingreso y gran parte de la población empobrecida.

5. Lento y retrasado proceso de incorporación de los avances científicos y tecnológicos.

6. Problemas de autosostenimiento alimentario.

7. Gran oferta de trabajo y escasez de demanda de trabajo y como conse- cuencia enorme presión del desemplec y subempleo.

\section{CONCLUSIONES Y RECOMENDA- CIONES}

El estudio de propuestas para el desarrollo nacional requiere de las cuentas nacionales como del PBI y población; pero estos estudios tienen que ser actualizados y departamentalizados para que complementados con una clara descripción de los principales problemas que el país enfrenta para el desarrollo, den pie a ideas $y$ soluciones. En este sentido, es muy positivo retomar la elaboración de planes con información actualizada.

Pero, no bastan los planes y la información. Es necesario canalizar las inquietudes y aspiraciones de las gentes de provincias, convertir su deseo en un proyecto técnicamente elaborado para que presente las bondades del mismo a los inversionistas, tanto nacionales como extranjeros bajo las mismas reglas de juego. Sólo así se podrá continuar la transformación de los ingentes recursos naturales para convertirlos en riqueza, en una primera etapa con intensa aplicación de mano de obra para dar empleo a la población, y que se visualice un sostenido proceso de reactivación económica, avance científico y tecnológico así como el logro del bienestar y progreso de la población peruana.

\section{BIBLIOGRAFÍA}

1 Congreso de la República. La Gaceta. Suplemento en el diario La República del 11 de agosto del 2002. Lima-Perú.

2 Instituto Nacional de Estadísticas e Informática. Perú; Compendio Estadístico 2001. Lima, junio del 2001.

4 Ministerio de la Presidencia. Planes Concertados de Gestión Estratégica Departamental. Lima, junio 2002.

5 Ministerio de la Presidencia. Estrategia de Desarrollo Territorial. Lima, julio 2002. 
3 Ministerio de la Presidencia. El PBI en el Perú. Descifra N. ${ }^{\circ} 2$, Secretaría Técnica para el Proceso de Descentralización. Lima, junio 2002.

4 Ministerio de la Presidencia. Planes Concertados de Gestión Estraté- gica Departamental. Lima, julio 2002.

5 Ministerio de la Presidencia. Estrategia de Desarrollo Territorial. Lima, julio 2002. 